\title{
Research Paper: \\ Comparison of the Effect of Custom Insole With CAD-CAM and Conventional Insole on FAOS Questionnaire Subscales in Patients With Plantar Fasciitis
}

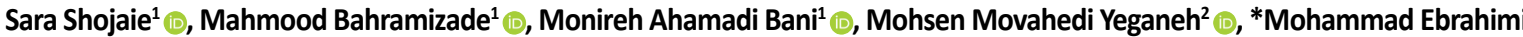 \\ Moosavi ${ }^{1}$ (i)
}

1. Department of Orthotics and Prosthetics, School of Rehabilitation Sciences, University of Social Welfare and Rehabilitation Sciences, Tehran, Iran. 2. Department of Orthopedic and Trauma Surgery, Milad Hospital, Tehran, Iran.

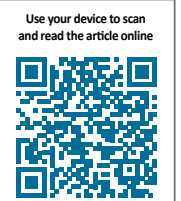

citation Shojaie S, Bahramizade M, Ahamadi Bani M, Movahedi Yeganeh M, Ebrahimi Moosavi M. [Comparison of the Effect of Custom Insole With CAD-CAM and Conventional Insole on FAOS Questionnaire Subscales in Patients With Plantar Fasciitis (Persian)]. Archives of Rehabilitation. 2020; 21(2):256-271. https://doi.org/10.32598/RJ.21.2.3052.1

doi https://doi.org/10.32598/RJ.21.2.3052.1

Received: 19 Jun 2019 Accepted: 09 Dec 2019 Available Online: $01 \mathrm{Jul} 2020$

Keywords:

Plantar fasciitis, CAD-

CAM, Insoles, Pain,

Quality of Life

\section{A B STR ACT}

Objective One of the significant causes of foot pain is plantar fasciitis. The use of medical insoles is a conventional treatment for this condition. The purpose of this study was to compare the effect of the customized insole with the CAD-CAM and conventional insole on pain, symptoms, daily activity, exercise and recreational activity, and quality of life in patients with PF.

Materials \& Methods This quasi-experimental study was performed on 14 patients with plantar fasciitis (five women and nine men) with the mean age of 40 years. Fourteen patients have been diagnosed with orthopedic pain after being diagnosed with plantar fasciitis according to inclusion criteria. Diagnosis of the complication of plantar fasciitis by orthopedic physician, Patients with flexible flat foot. No neurological disorders or any foot pathology such as diabetes and osteoarthritis. Patients were randomly assigned into two groups of 7 using customized insole with CAD-CAM and conventional (prefabricated) insole. From the outset it was found to be one of two types of insole to be studied: first the patient was given a CNC insole, and the other 13 patients received the same insole and divided into two groups. The instrument of this study was the FAOS questionnaire (foot and ankle outcomes) which measures the five variables of pain, symptoms, daily activities, sports and recreational activities, and quality of life. Both groups completed the questionnaire before using the insole and again after six weeks. Compressive scan of both groups was accomplished using EMED foot pressure system. The custom-made insole was designed using Rhino Cross software and then shaved using EVA foam blocks with $50 \%$ shore using a CNC machine. In the conventional insoles group, patients received conventional polyurethane insoles based on the length measurements of the single leg. Patients completed the questionnaire again after six weeks. Data were analyzed by SPSS software v. 22. After checking the normality of the data by Shapiro-Wilk test, non-parametric Mann-Whitney and Wilcoxon tests were used to analyze the data.

Results In the pre-intervention phase, there was no significant difference in pain, symptoms, daily activities, recreational-sports activities, and quality of life in the two groups, and the two groups were homogeneous. After six weeks, there was a significant difference between the two groups in the FAOS questionnaire $(\mathrm{P}<0.05)$. There was no significant difference between the two groups in pain score, symptoms, daily activities, recreational-sport activities, and quality of life $(P<0.05)$.

Conclusion According to the results of this study, both customized insoles with CAD-CAM and conventional insoles are effective in improving FAOS questionnaire subscales. There was no significant difference in the impact between the two types of insoles in foot and ankle.

\section{* Corresponding Author:}

Mohammad Ebrahimi Moosavi, PhD.

Address: Department of Orthotics and Prosthetics, School of Rehabilitation Sciences, University of Social Welfare and Rehabilitation Sciences, Tehran, Iran.Tel: +98 (21) 22180010

E-Mail: e.mousavi2001@gmail.com 


\section{Extended Abstract}

\section{Introduction}

$\mathbf{P}$

lantar fasciitis is defined as inflammation or destructive changes in the plantar fascia. Pain and stiffness in the soles of the feet are often a common symptom of the disease, which is often felt when standing after a long period of sitting or sleeping. This complication has a negative effect on foot function and limits a person's daily activities [1, 2]. In general, $10 \%-15 \%$ of people in the world suffer from plantar fasciitis $[1,2]$.

Surgical and non-surgical treatments are available for this complication which among non-surgical interventions, orthotic (protective) treatments are widely used to treat plantar fasciitis [3]. Among orthotic treatments, custommade insoles and prefabricated insoles are significantly prescribed in the treatment of plantar fasciitis [4]. Both custom-made and prefabricated insoles are effective in reducing pain and increasing patient function, but there are conflicting studies about the superiority of custom-made insoles and prefabricated insoles [5].

In these studies, the effect of custom insole with Computeraided Design-Computer-aided Manufacturing (CAD-CAM) device in the treatment of patients with plantar fasciitis was not investigated [6]. In a review study, it was shown that computer insole fabrication is a valid method that can be used in the field of medical insole fabrication to achieve the best fit between the insole and the patient's foot [7]. Custommade medical insole is a factor that is directly related to the proper placement of the insole in the sole of the foot and is associated with reducing the amount of damage to the sole of the foot [8]. The main purpose of using this type of medical insole is to change the pressure distribution of the sole of the foot from a specific point to a wider surface $[9,10]$.

Insoles made by CAD-CAM method reduce the force applied to high pressure areas through full contact with increasing the level and it was shown during the studies that the mentioned insole reduced the pressure of high-risk points for diabetic wounds [11]. Recent studies have also shown that computer-made insoles reduce pain in people with flat feet [12]. Another study that examined the effect of custommade insoles made by CAD-CAM method in people with pes cavus foot, the use of this insole was said to reduce the pain of these people due to the proportional distribution of pressure and force distribution from the heel [13].

Considering the reviewed studies and the significant prevalence of plantar fasciitis and its negative impact on patient performance and activity and due to the fact that in these studies, the effect of the insole made by CAD-CAM method in people with plantar fasciitis has not been studied and Reports of other complications, such as diabetes and flat feet, suggest that custom-made insoles distribute pressure evenly across high-risk areas, therefore, in this study, we reviewed and compared the custom insole made by computer method with the prefabricated insole [14].

\section{Materials and Methods}

This quasi-experimental study was performed in 2018 in the medical centers of Tehran University of Social Welfare and Rehabilitation Sciences on 14 patients with plantar fasciitis (five women and nine men) with an average age of 40 years. The inclusion criteria of the research were being aged 25-45 years, diagnosis of plantar fasciitis by an orthopedist, existence of flexible flatfoot complication, no neurological disorders or any pathology in the foot such as diabetes and osteoarthritis and lack of surgery on the lower limb joints in the past month and the ability to walk independently without an aid [11, 15-18]. Exclusion criteria were people with a history of surgery on the plantar fasciitis and patients were also excluded from the study if insoles were not used during the study and if they were professional athletes [18].

The tool used to measure the variables of symptoms, pain, daily activities, sports and recreational activities and quality of life before and after the test was the Foot and Ankle Outcome Score (FAOS) questionnaire. The scoring of this questionnaire was from 0 to 100 , which 0 refers to the worst situation and 100 refers to the best situation.

After the diagnosis of plantar fasciitis by an orthopedist and the evaluation of the patient by an orthopedist and prosthetist based on the reported history (for example, the first step is accompanied by pain in the leg area), clinical tests (such as local pain when touched in the anterior-internal part of the heel tuberosity) or pain during plantar fasciitis of patients if they meet the inclusion criteria to enter the study, 14 patients were assigned by non-probability simple (available) method and were randomly divided into two groups of 7 using custom insole with CAD-CAM device and using conventional (prefabricated) insole group. Patients were initially given a FAOS questionnaire to determine their pain, symptoms, daily activities, sports and recreational activities, and quality of life before using the insole. Then, in both groups, the necessary measurements were performed on each person's foot. The map of each person's foot was taken in equal weight bearing condition on both feet and the patients were scanned by EMED Foot Pressure scanner [19]. 


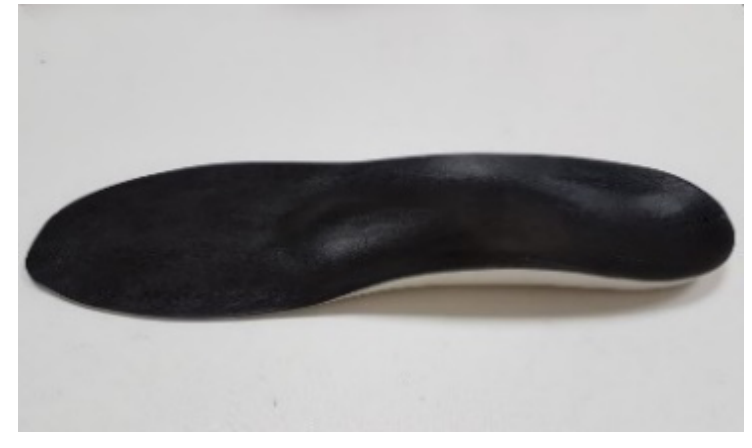

Figure 2. Prefabricated insole

In the group of patients selected to make custom insoles for by CAD-CAM method, after taking a compression scan of the patient's foot, the compression scan information of each patient was evaluated separately. Then, based on the scan report in these patients, a custom-made insole was designed for each patient separately using Rhinoceros computer software by the therapist. In the next step, the data obtained from the design in the form of a set of codes called G-code, was given to the CNC (Computer Numerical Control) machine made by Paya-Fanavaran Company and as can be seen in Figure 1, the medical insole was shaved on a foam block made of ethyl vinyl acetate with $50 \%$ saline. The height of the longitudinal-internal arch in the custom-made insole was considered to be $15 \mathrm{~mm}$ [20]. The length of the medial longitudinal arch was also calculated based on the size of the patient's foot from the talus to the first metatarsus [21]. The width and length of the metatarsal pad were also based on a study by Hastings et al. [10] and based on the foot size of each patient, the length of the metatarsal pads were chosen to be between $65-75 \mathrm{~mm}$ and their width were between $63-51 \mathrm{~mm}$ and their height were between $9-11 \mathrm{~mm}$ and they were made based on these dimensions [22].

In the second group, conventional insoles were prepared based on the measurement of each person's foot. The mentioned prefabricated insole is made of polyurethane and has a longitudinal-inner arch and metatarsal pad (Figure 2). Patients in both groups were then asked to put insoles inside their shoes. To avoid discrepancies in shoes, patients were asked to provide shoes with laces and appropriate size that have a suitable space in the front of the foot and after the patients' shoes were approved by the therapist, both groups of patients were told to use insoles for 7-10 hours every day while working and walking outdoors for 6 weeks. At the end of the treatment period, the patients were given the questionnaire again to re-report the studied variables. Then, we calculated the score related to each of the sub-scales of the questionnaire according to its formula in the questionnaire guide, before using the insole and after using the insole for each individual.

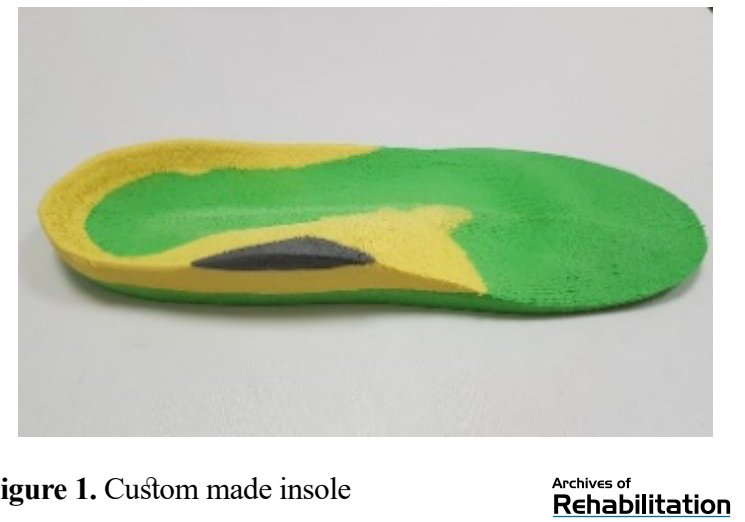

The final scores of both groups at the time before the study and also 6 weeks after the study, which were obtained from the questionnaires completed by individuals, were entered into SPSS software V. 22. After calculating the mean scattering indices and standard deviation and measuring the normality of the data using Shapiro-Wilk, Mann-Whitney $\mathrm{U}$ and Wilcoxon tests were used to evaluate the data.

\section{Results}

The study was performed on 14 patients with plantar fasciitis who referred to the clinic that half of them were studied by conventional insole and the other half by custom insole made using CAD-CAM device. The Mean $\pm \mathrm{SD}$ age of patients in the custom-made insole group was $40.14 \pm 5.98$ years and the Mean $\pm \mathrm{SD}$ of patients in the conventional insole group was $37.86 \pm 5.58$ years. The Mean $\pm \mathrm{SD}$ of BMI of patients in the custom-made insole group was $26.07 \pm 1.76$ and the Mean \pm SD of BMI of patients in the conventional insole group was 27.04 \pm 2.46 .

First, the 5 main factors of FAOS questionnaire including pain, symptoms, daily activities, sports and recreational activities, and quality of life in patients with plantar fasciitis were evaluated separately in both types of insole groups and finally, we compared all the factors between the two insole groups. The scoring of FAOS questionnaire factors was such that the higher the score, the more desirable and the lower, the more undesirable.

According to Table 1, which is the result of Wilcoxon statistical test, it can be seen that the rate of improvement of pain, symptoms, daily activities, sports and recreational activities, and quality of life before and after 6 weeks of using custom-made insoles was significant.

According to Table 2, which is the result of Wilcoxon statistical test, it can be seen that the rate of improvement of pain, symptoms, daily activities, sports and recreational ac- 
Table 1. Evaluation of the scores of the subscales evaluated in patients of the custom-made insole group

\begin{tabular}{cccc}
\hline \multirow{2}{*}{ Custom-made Insole } & \multicolumn{2}{c}{ M ean \pm SD } & $\begin{array}{c}\text { Significance Level Before and After } \\
\text { Intervention }\end{array}$ \\
\cline { 2 - 4 } & Before Intervention & After Intervention & 0.018 \\
\hline Pain & $50 \pm 20.7$ & $71.03 \pm 16.96$ & 0.028 \\
\hline Symptoms & $45.92 \pm 10.6$ & $58.3 \pm 16.96$ & 0.018 \\
\hline Daily activities & $56.51 \pm 22.65$ & $75.63 \pm 15.18$ & 0.018 \\
Sports and recreational activi- & $33.57 \pm 28.97$ & $62.14 \pm 20.18$ & 0.038 \\
\hline ties & $38.39 \pm 26.86$ & $44.64 \pm 28.05$ & \\
\hline Quality of life & &
\end{tabular}

Archives of

Table 2. Evaluation of scores of subscales evaluated in patients of conventional insole group

\begin{tabular}{|c|c|c|c|}
\hline \multirow{2}{*}{ Custom-Made Insole } & \multicolumn{2}{|c|}{ Mean \pm SD } & \multirow{2}{*}{$\begin{array}{l}\text { Significance Level Before and } \\
\text { After the Intervention }\end{array}$} \\
\hline & Before Intervention & After Intervention & \\
\hline Pain & $69.44 \pm 14.25$ & $81.34 \pm 11.08$ & 0.017 \\
\hline Symptoms & $47.96 \pm 13$ & $59.18 \pm 7.95$ & 0.05 \\
\hline Daily activities & $71 \pm 18.94$ & $87.39 \pm 11.42$ & 0.018 \\
\hline Sports and recreational activities & $49.29 \pm 30.74$ & $73.57 \pm 14.35$ & 0.018 \\
\hline Quality of Life & $32.14 \pm 15.06$ & $45.54 \pm 11.81$ & 0.027 \\
\hline
\end{tabular}

Rehrchives of

Table 3. Comparison of the evaluated subscales between the two groups after 6 weeks

\begin{tabular}{cc}
\hline Subscale & Significance Level After Intervention Between the Two Groups \\
\hline Pain & 0.0128 \\
Symptoms & 0.902 \\
Daily activities & 0.710 \\
Sports and recreational activities & 0.535 \\
\hline Quality of Life & 0.209 \\
\hline
\end{tabular}

Rehabilitation

tivities and quality of life before and after 6 weeks of using the conventional insole is significant.

According to the result of Mann-Whitney test in Table 3, we see that in the rate of improvement of pain, symptoms, daily activities, sports and recreational activities and quality of life after 6 weeks of using both types of custom insoles using CAD-CAM and conventional insoles, no significant differences were shown.

\section{Discussion}

The results of the present study were in line with the study of Bonanno et al. [23]. They stated that the prefabricated insole distributes the force evenly over a wider area due to full contact with the patient's sole [24]. Full-length insoles with longitudinal-internal arch reduce these forces further by reducing the vertical forces on the heel and due to the wider surface, they create, and as a result, the pain in this area is reduced [25]. 
Our study was in line with the study of Michelle Drake [26]. The author stated in this study that custom insoles reduced tensile stress in plantar fascia due to sufficient longitudinal-internal arch height $[27,28]$ and as a result, pain and disability were significantly reduced [26].

The result of the present study was in line with the result of Burns study [13]. In the Burns study, a significant improvement in pain was reported in a group of patients with pes cavus. The orthosis used in this study was a custom insole using the CAD-CAM system [13], insoles made with CAD-CAM system distributed pressure peak and maximum force distribution from the heel to the inner part of the midfoot area and were used to reduce the maximum pressure concentration at a specific point [14]. Because the sole of the foot is the heel and the removal of pressure from this area helps to improve pain and increase the function of patients with the sole of the foot, it is likely that the custom insole made by our study also distributes the maximum pressure from the heel.

The reduction of pain observed after using both insoles in the present study may also be due to the support of the longitudinal-internal arch of the foot by the insole, including the heel area and also the support of the transverse arch of the foot by the metatarsal pad. According to a Burns study in 2005, these three factors also caused a proportional distribution of plantar pressure and reduced load applied to the heel area, followed by pain in this area [29].

The result of the present study was inconsistent with the study of Pfeffer Glenn; in Glenn's study, the silicone heel as a prefabricated insole caused more pain in the heel area than the custom insole made in this study, which was made of polypropylene [30]. The author stated that if the custom-made insole used more shock-absorbing material, it might have worked better than the propylene insole to reduce pain [31]. In the study, the author considered the use of soft and shock-absorbing materials as the reason for the superiority of silicone heels and stated that the propylene used is a hard material. Of course, the custom insole made in our study was made of EVA and had less shore than polypropylene (shore $=70$ ) [32].

In the field of daily activities and sports and recreational activities of patients with plantar fasciitis, the custom insole made the study by Oliveira et al. more comfortable because it matched the patient's foot and due to the use of soft foam in the heel area, it was more comfortable for this part [6]. Based on the results obtained in this study, the insole reduced the forces applied to the heel and subsequently reduced the pain and improved performance [26]; we can say that a person's activity increases due to the reduction of heel pain. Also, the insole we studied improves the performance and thus increases the activity of the person for the same reasons as the study of Oliveira.

In a study by Roos et al., A custom insole was reported to be a better choice in the treatment of plantar fasciitis and was able to increase the level of performance of people with plantar fasciitis compared to nocturnal splints [4]. In a study by Drake Michelle et al., using a custom insole for 2 weeks improved the performance and ability of patients with plantar fasciitis [26]. In a study by Drake Michelle et al., using a custom insole for 2 weeks improved the performance and ability of patients with plantar fasciitis and it was reported that using the insole with daily stretching improved its effects. In a study by Burns et al., Custom-made orthoses were able to increase the level of performance of people with pes cavus [13]. Novak et al. Also concluded that functional orthoses compared to flat insoles (without arch) had a significant effect on increasing the function of patients with rheumatoid arthritis [33].

The results of the present study were consistent with the studies mentioned above. In the prefabricated insole of the present study, due to the presence of the longitudinal-internal arch and the inclusion of the entire surface of the foot, it causes the distribution of force on a larger surface, followed by removal of pressure from the heel area (which is the source of the sole). In addition to all the items mentioned in the prefabricated insole, the softer foam in the substrate can be mentioned in the custom insole made by the patients in this case, which creates a better fit.

Regarding the quality of life of plantar fasciitis patients, Ross et al. measured the effect of insoles and night splints in people with plantar fasciitis and in both groups, an improvement in quality of life was observed [4]. In the Burns study, orthoses improved the quality of life of people with pes cavus and there was a significant difference in the quality of life variable between the group that used orthoses and the control group [13]. In his study, Burns compared a study group that had a custom insole made using the CAD-CAM system with another group that did not have an insole (a simple placebo insole) and therefore, it can be said that it is similar to the present study and their results are in one direction.

\section{Conclusion}

Both insoles were effective in improving all subscales of the FAOS questionnaire (pain, symptoms, daily activities, sports and leisure activities, and quality of life) after use for six weeks, but comparing the two insoles numerically, despite The custom insole showed more improvement using the CAD-CAM device than the conventional insole in most 
subscales, but there was no statistically significant difference between the two insoles and both insoles improved foot and ankle outcomes. Therefore, due to the lack of significant differences between the two types of insoles and their effectiveness in improving the variables mentioned in the treatment of patients, this study suggests the use of both types of insoles in medical centers.

\section{Ethical Considerations}

Compliance with ethical guidelines

This study was approved by the University of Social Welfare and Rehabilitation Sciences Medical Center. The principles are considered in this article. The participants were informed about the purpose of the research and its implementation stages; they were also assured about the confidentiality of their information; moreover, they were free to leave the study whenever they wished, and if desired, the research results would be available to them.

\section{Funding}

The present paper was extracted from the MSc. thesis of the first author, Department of Orthotics and Prosthetics, School of Rehabilitation Sciences, University of Social Welfare and Rehabilitation Sciences.

\section{Authors' contributions}

All authors contributed in preparing this article.

\section{Conflicts of interest}

The authors declared no conflict of interest. 
This Page Intentionally Left Blank 
مقاله بروهشى

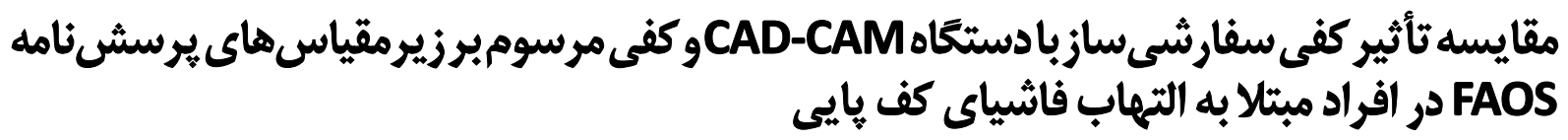

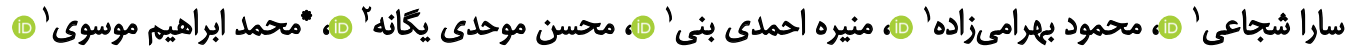
ا.كروه ارتويدى و اعضاى مصنوعى و وسايل كمكى، دانشكاه علوم بهزيستى و توانبخشى، تهران، ايران. r. كروه جراحى ارتويدى و تروما، بيمارستان ميلاد، تهران، ايران.

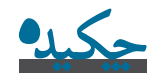

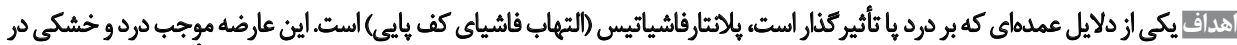

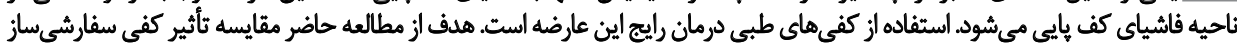

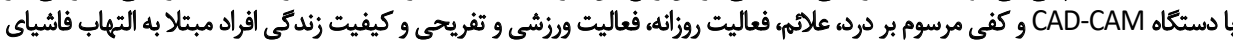
كفي باييى است.

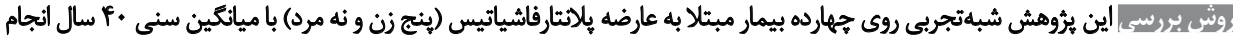

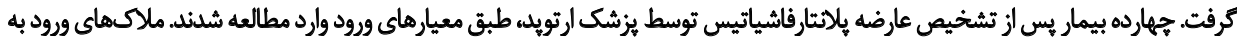

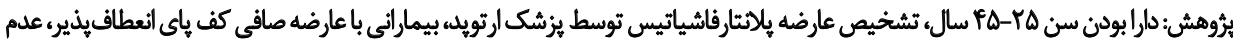

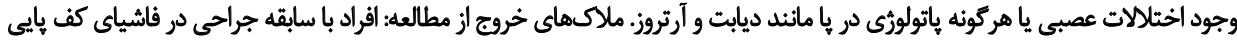

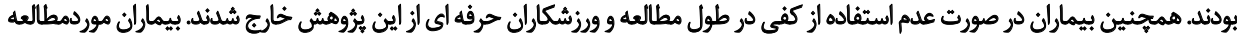

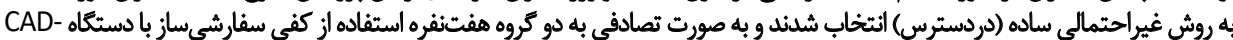

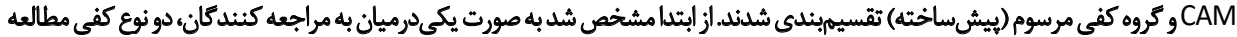

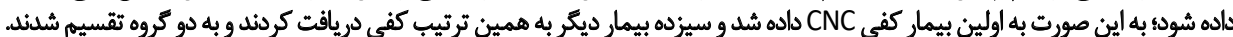

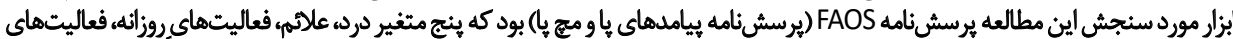

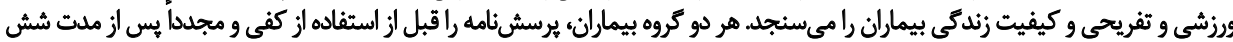

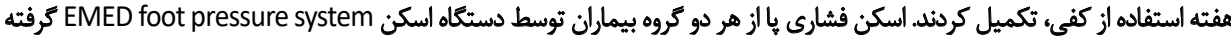

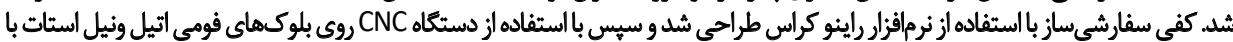

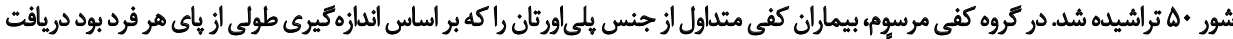

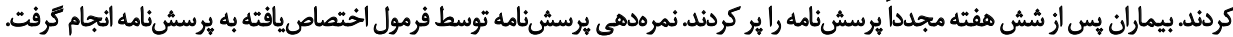

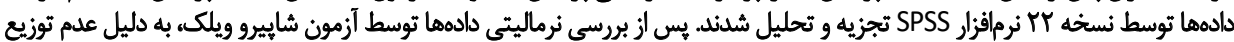

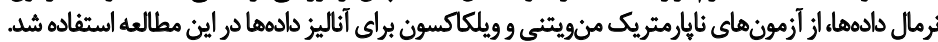

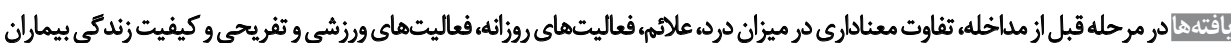

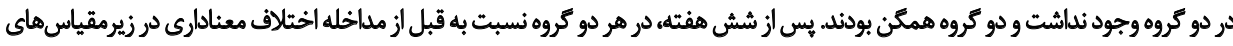

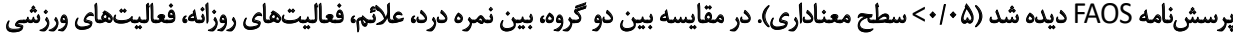

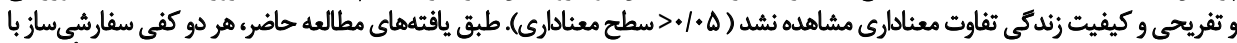

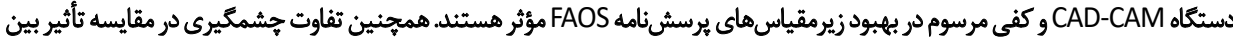

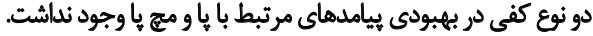

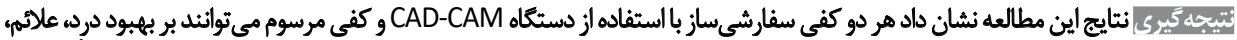

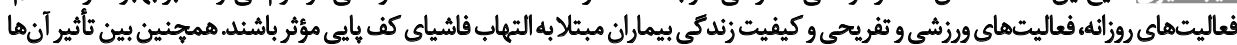

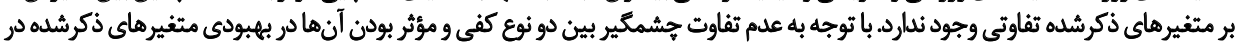

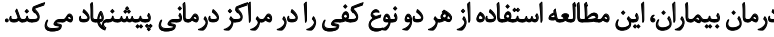

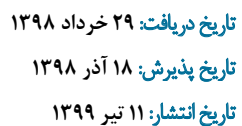

كليدوازٔها:

التهاب فاشياى كف

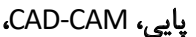
كفي، درد، كيفيت زندكى 
"اشنه براي كاهش فشار در ناحيه ياشنه كزارش كرده بودند [N]].

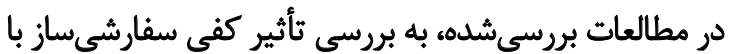

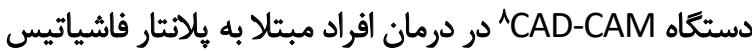

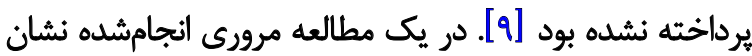

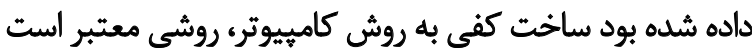

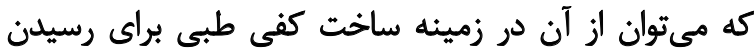

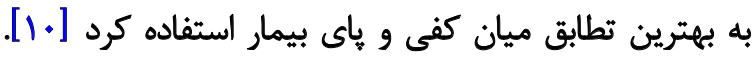

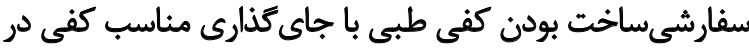

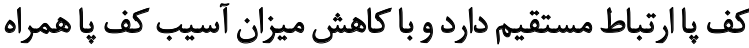

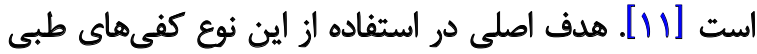

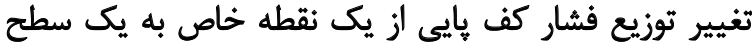

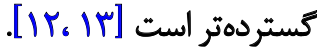

كفى هاى ساختهشده به روش CAD-CAM از طريق تماس

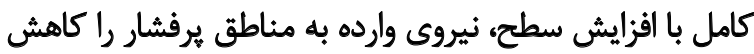

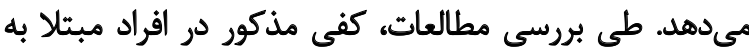

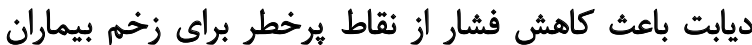

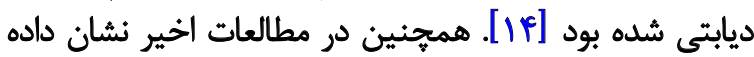

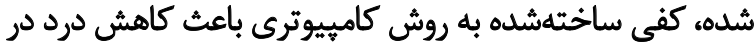

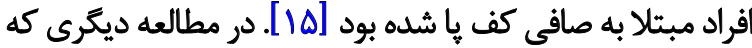

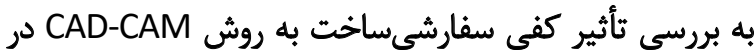

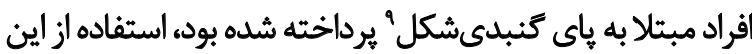

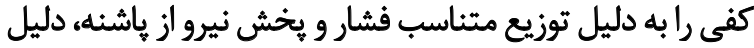

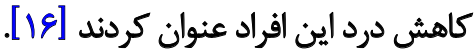

بادر نظر كرفتن مطالعات بررسى إشده وشيوع قابل توجه بيمارى

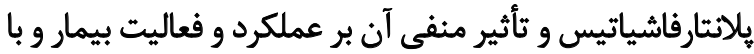

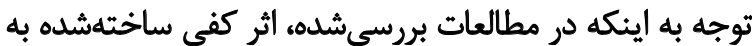

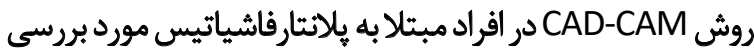

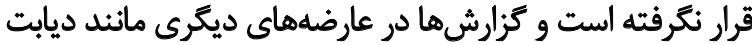

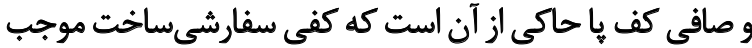

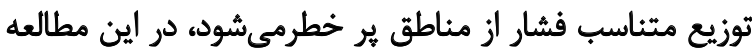

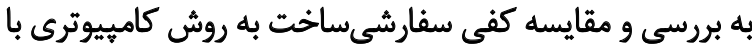

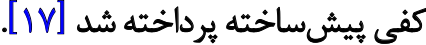

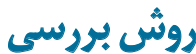

اين يُؤهش شبهتجربي در سال لو إ در مراكز درمانى دانشكاه

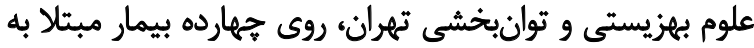

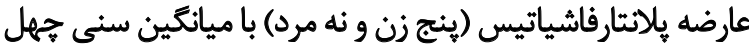

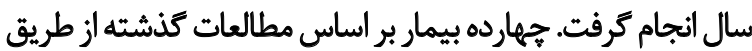

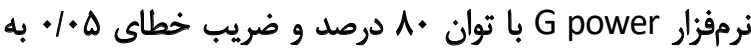

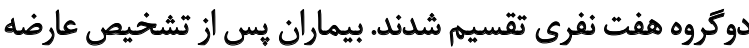

8. Computer-aided Design / Computer-aided Manufacturing 9. Cavus Foot
مقام

يلانتارفاشياتيس' به عنوان التهاب و يا تغييرات تخريبى در

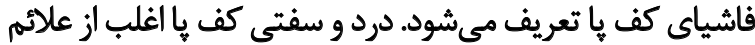

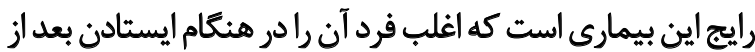

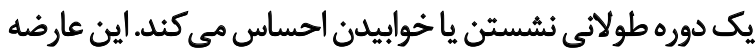

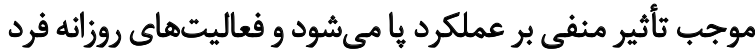

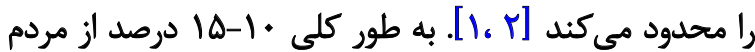

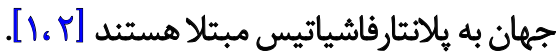

درمانهاى جراحى وغير جراحى براى اين عارضه موجود هستند

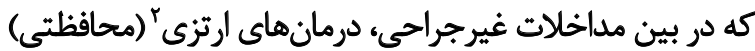

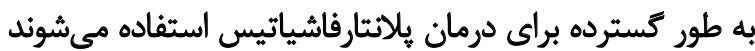

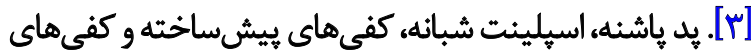

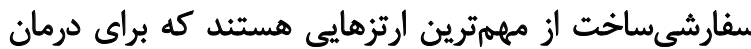

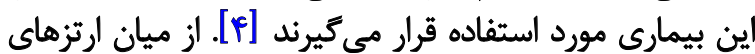

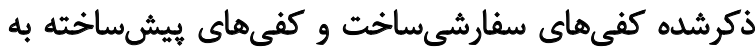

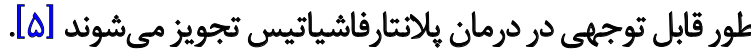

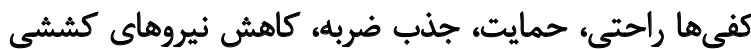

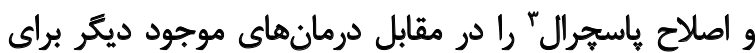

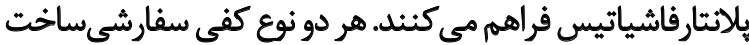

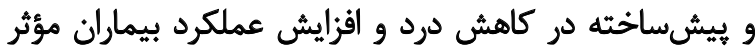

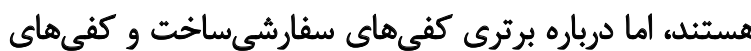

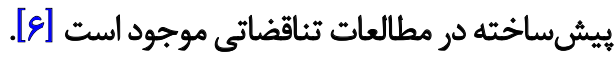

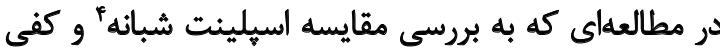

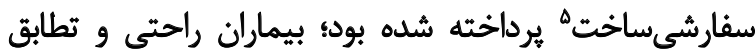

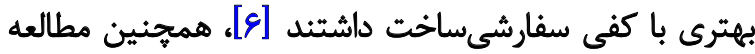

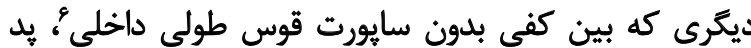

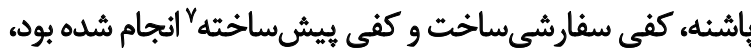

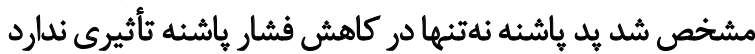

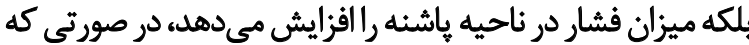

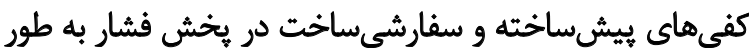

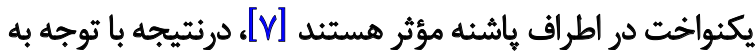

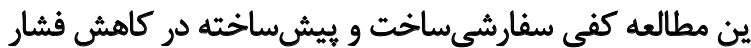

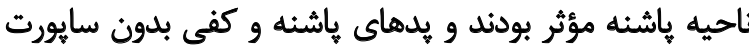

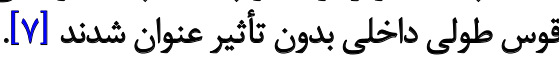

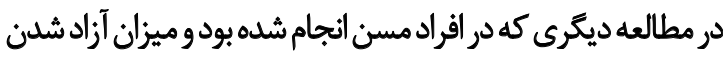

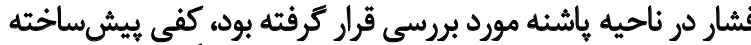

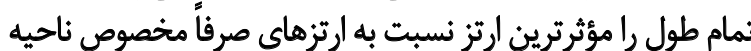

1. Plantar Fascitiis

2. Conservative

3. Postural

4. Night splint

5. Custom insole

6. Flat

7. Prefabricated 
از كفى سفارشى ساز با دستكاه CAD-CAM و كروه كفى مرسوم

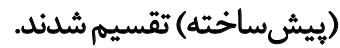

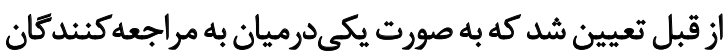

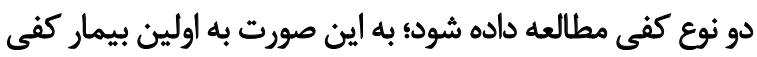

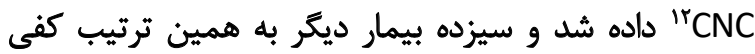

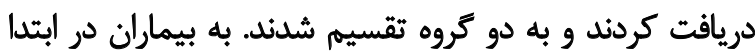

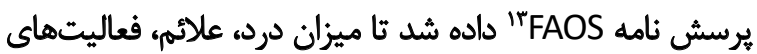

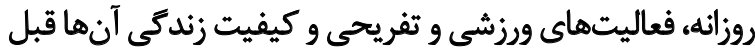

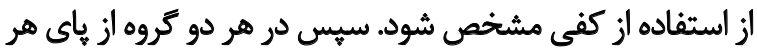

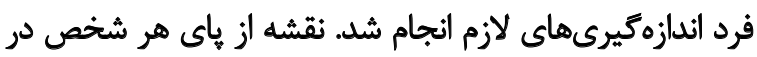

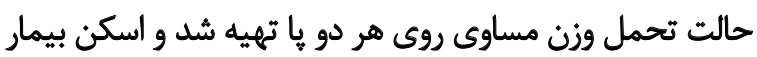

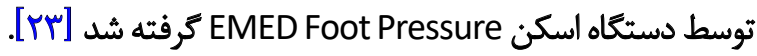

در زروه بيماران انتخابشده براى ساخت كفى سفارشى باخت

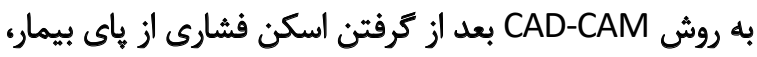

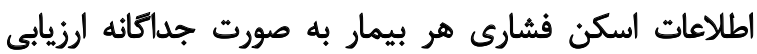

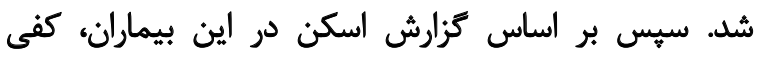

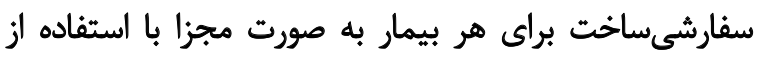

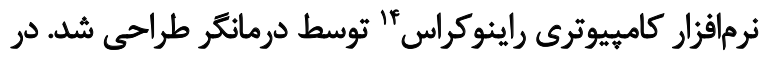

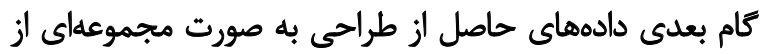

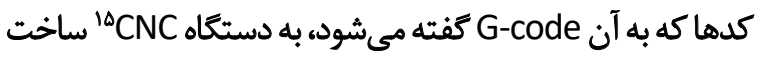

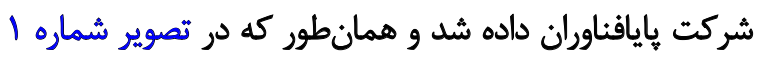

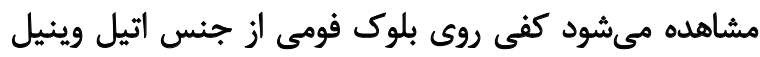

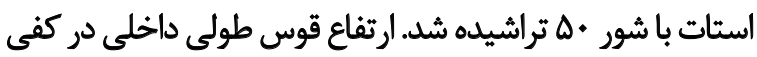

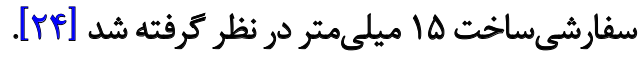

همجنين طول قوس طولى داخلى بر اساس اندازه بإى بيمار

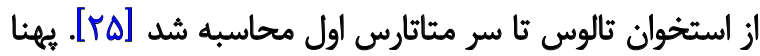

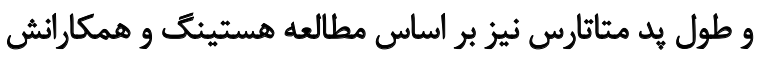

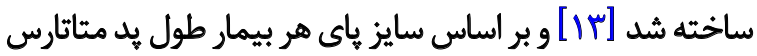

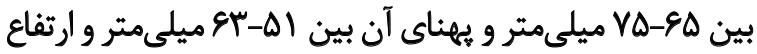

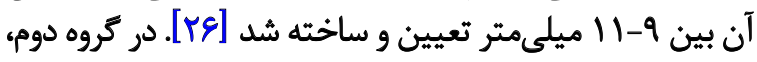

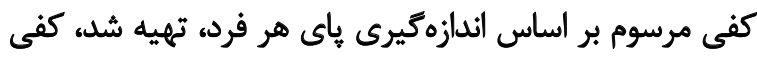

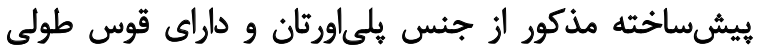
داخلى و يد متاثارس بود (تصوير شماره جان).

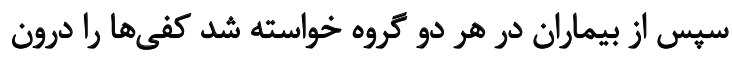

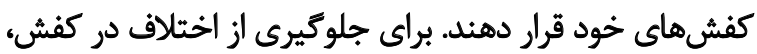

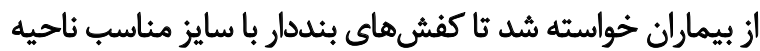

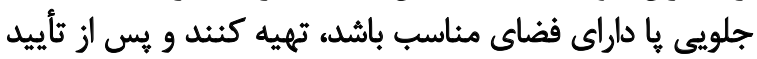

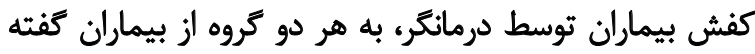
شد شش هفته و هر روز هفت تا ده ساعت در هنكام درام فعاليت

12. Computer Numerical Control

13. Sport and Recreation

14. Rhinoceros 5

15. Computer Numerical Control
هيلانتارفاشياتيس توسط يزشك ارتويد، طبق معيارهاى ورود وارد

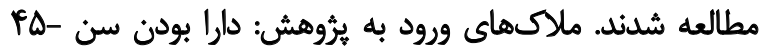

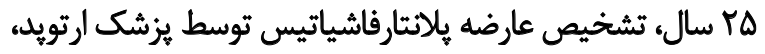

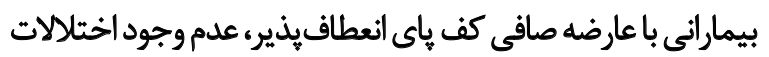

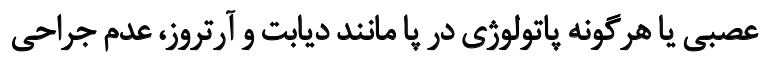

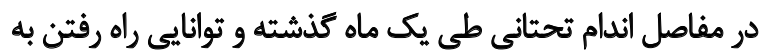

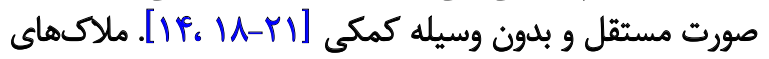

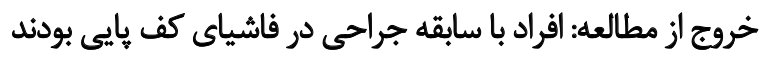

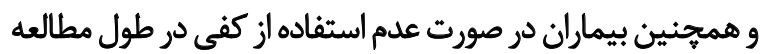

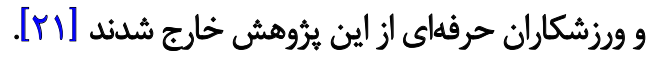

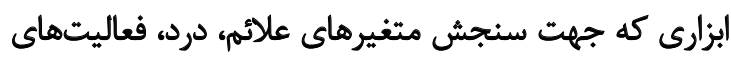

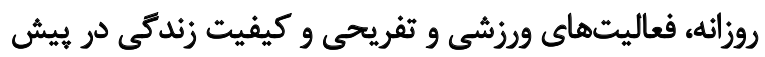

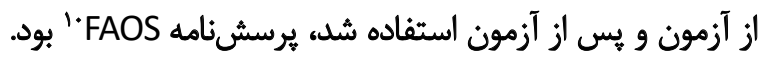

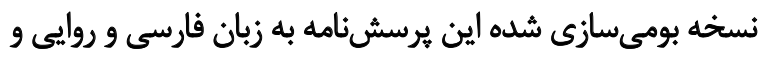

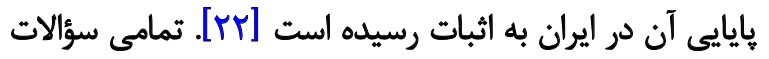

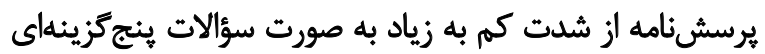

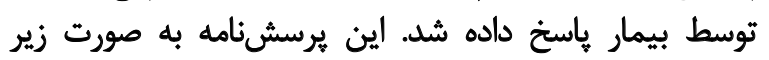

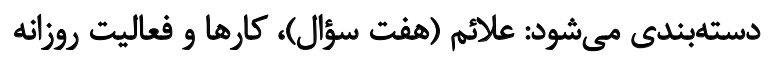

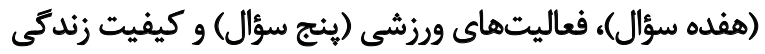

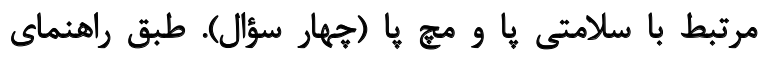

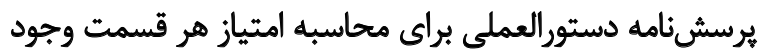

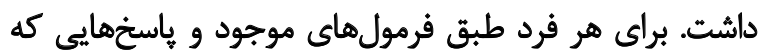

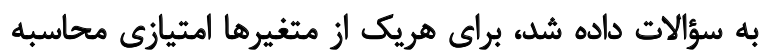

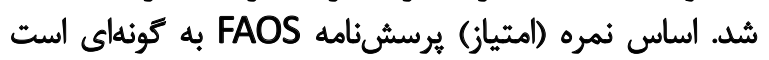

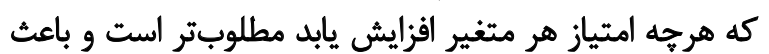

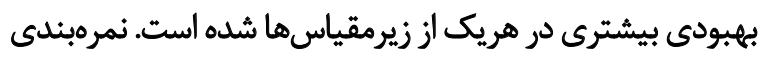

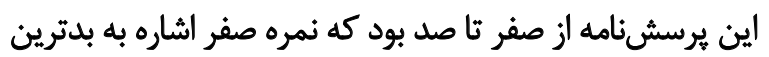

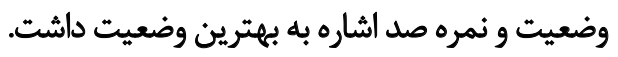

\section{روش جمع آورى اطلاعاث}

يس از تشخيص عارضه هيلانتارفاشياتس توسط بزشك ارتوبيد

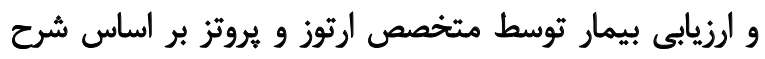

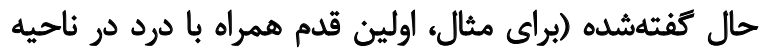

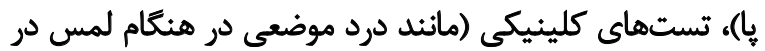

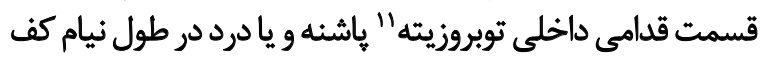

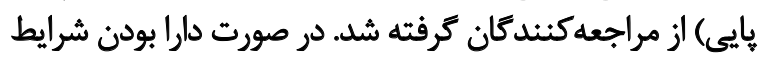

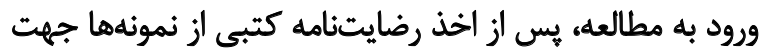

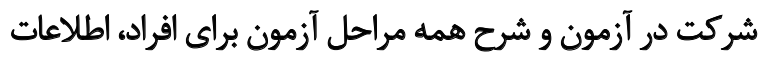

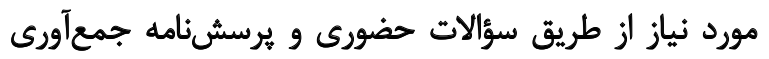

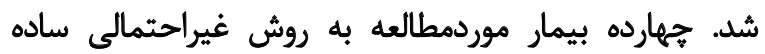
(دردسترس) به صورت تصادفى به دو مروه هفتنفره بهره استفادها

10. Foot and Ankle Outcome Scale

11. Tuberosity 


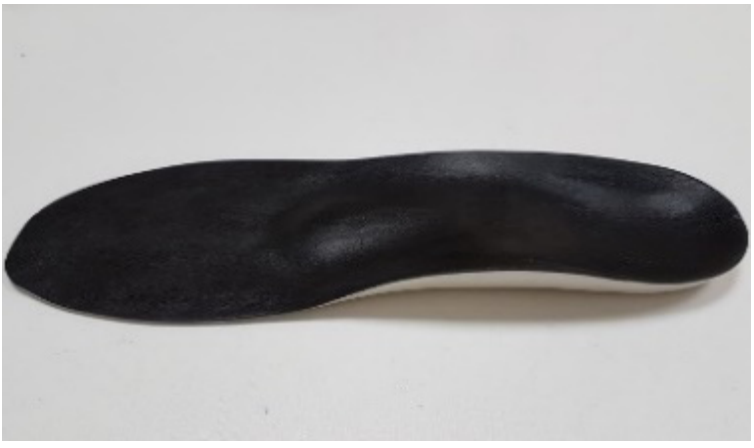

توانبخننى

$$
\text { تصوير r. كفى يُيشساخته }
$$

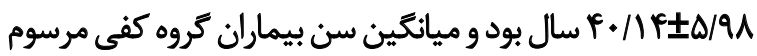

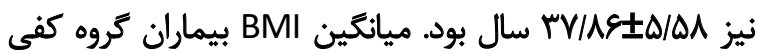

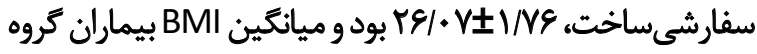
كفى مرسوم نيز

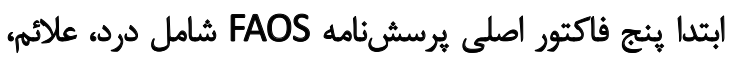

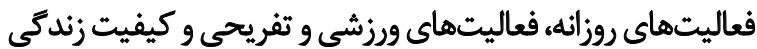

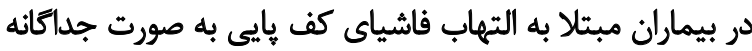

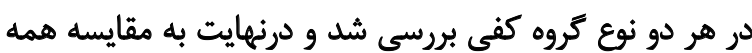

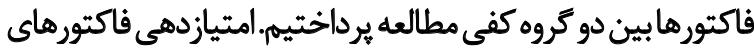

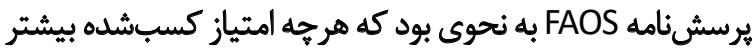

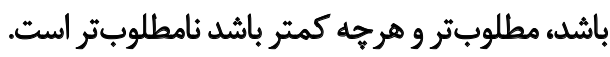
بر اساس جدول شماره إكه نتيجه آزمون آمارى ويلكاكسون

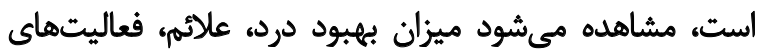

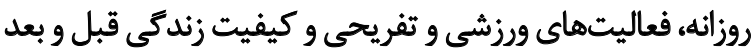
از شش هفته استفاده از كفى سفارشى وراخت معنى معنادار شده است بر اساس جدول شماره Y كه نتيجه آزمون آمارى ويلكاكسون

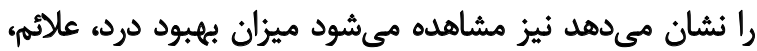

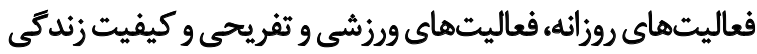
قبل و بعد از شش هفته استفاده از كفى مرسوم معنادار شده است با توجه به نتيجه آزمون آمارى منويتنى در جدول شماره

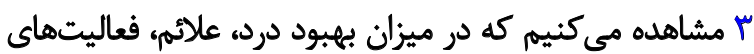

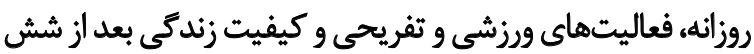

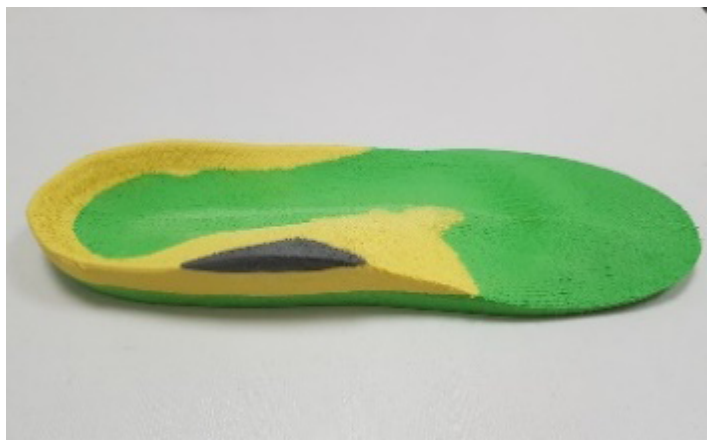

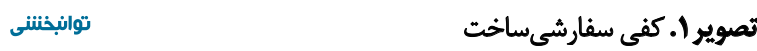

و راه رفتن در خارج از منزل از كفى استفاده كنند. در طول

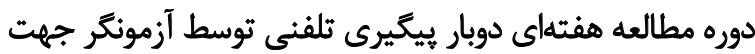

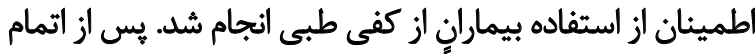

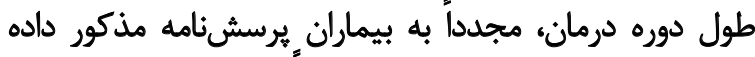

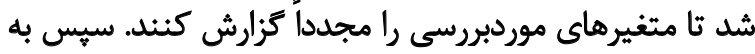

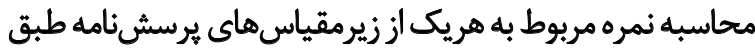

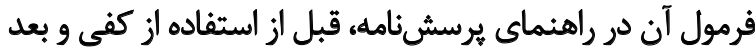

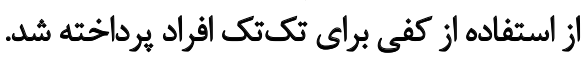

نمرات نهاييى هر دو گروه در زمان قبل از مطالعه و همجنين

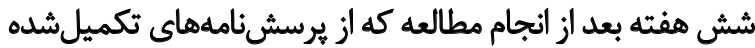

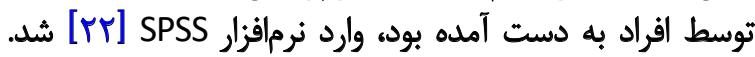

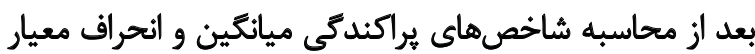

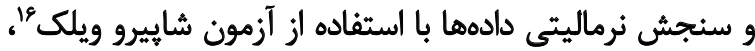
از آزمونهاى منويتنى و ويلكاكسون براي ارزيابى دادهها استفاده آناهي

Latrots

مطالعه روى جهارده نفر بيمار مبتلا به التهاب فاشياى كف إيايى

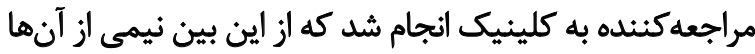

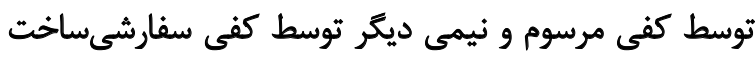

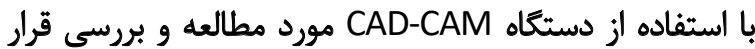

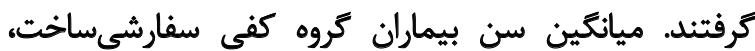

16. Shapirowilk-Test

جدول ا ب بررسى امتيازهاى زيرمقياسهاى موردارزيابى در بيماران كروه كفى سفارشىساخت

\begin{tabular}{|c|c|c|c|}
\hline سطح معنادارى قبل و بعد از مداخله & بعد از مداخله & قبل از مداخله & كفى سفارشى \\
\hline $.1 \cdot 11$ & $V W \cdot r \pm 1 E / Q$ & $\Delta \cdot \pm r \cdot N$ & 2,2 \\
\hline $.1 .4 A$ & $\Delta N r \pm 18 / Q C$ & $\mid \mathrm{F} / \mathrm{q} Y \pm 1 . / 8$ & مille \\
\hline .1 .11 & $V \Delta / / \mathscr{Q} \pm 1 Q / / \Lambda$ & $\Delta \& / \Delta) \pm Y Y / \& \Delta$ & فعاليتهاى روزانه \\
\hline $.1 * 11$ & $g r / I F \pm r+/ 1 R$ & rr/DVITNaY & فعاليت ورزشى و تفريحى \\
\hline $.4 \mathrm{r}$ & 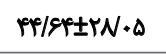 & TNMATKEN & كيفيت زندكى \\
\hline
\end{tabular}

توانبخننى 


\begin{tabular}{|c|c|c|c|}
\hline \multirow{2}{*}{ سطح معنادارى قبل و بعد از مداخله } & \multicolumn{2}{|c|}{ ميانكين+انحراف معيار } & \multirow[t]{2}{*}{ كفى مرسوم } \\
\hline & بعد از مداخله & قبل از مداخله & \\
\hline.$/ 18$ & $A / / M \pm \pm 11 / * A$ & $E q / F+ \pm I F / T Q$ & درد \\
\hline 1.0 & $\Delta \vee / \Lambda \pm V / q \Delta$ & Pr/atIr & عالائم \\
\hline$\% 14$ & $A V / T a \pm I I / P T$ & nEINar & فعاليتهاي روزانه \\
\hline $.1 \cdot 11$ & $V T / \Delta V \pm I f / T \Delta$ & $r q / P q \pm r \cdot / M^{*}$ & قعاليت ورزشى و تفريحى \\
\hline$\% t r$ & $F \Delta / \Delta f \pm|1 / A|$ & $M / / 1 \pm \pm \mid Q / \cdot 9$ & كيفيت زندكى \\
\hline
\end{tabular}

جدول ". مقايسه زيرمقياسهاى موردازيابى بين دو كروه بعد أز شش هفته

\begin{tabular}{|c|c|}
\hline سطح معناهارى بعد از مداخله بين دو كروه & زيرمقياس \\
\hline.$/ .1 T A$ & درد \\
\hline$. / 9 . r$ & علاثيم \\
\hline$\cdot M$ & فعاليتهايى روزأنه \\
\hline . lara & فعاليت ورزشى و تفريحى \\
\hline$+\pi+9$ & كيفيت زندكى \\
\hline
\end{tabular}

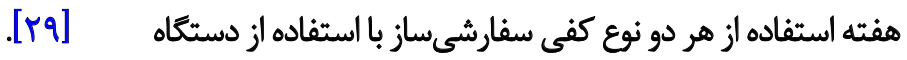

نتيجه مطالعه حاضر با نتيجه مطالعه برنز نيز همسو بود [1 [1]

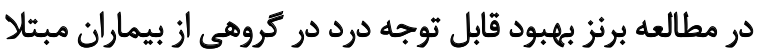

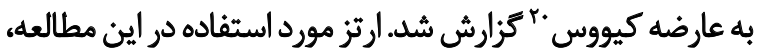

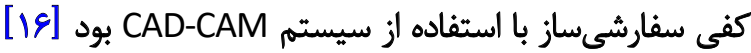
كفىهاى ساختهشده با سيستم

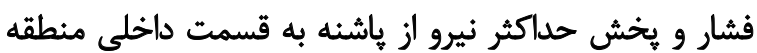

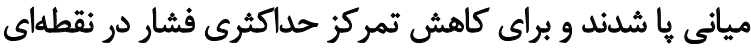

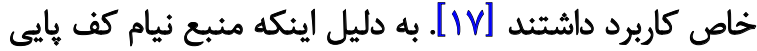

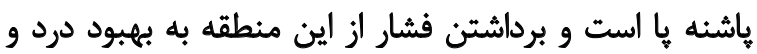

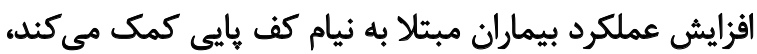

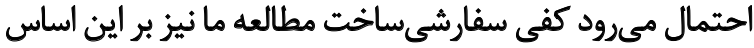
موجب يخش حداكثر فشار از ياشنه مىشئرد

احتمال ميرود كاهش درد مشاهدهشده بعد از استفاده از هر دري

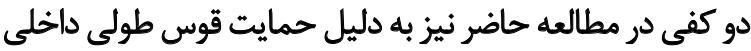

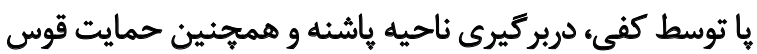

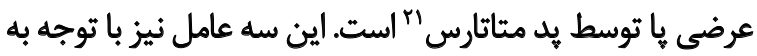

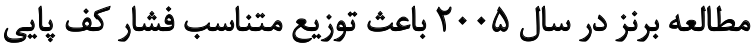

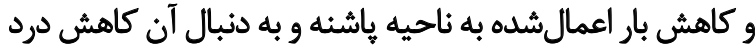

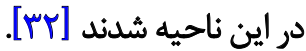

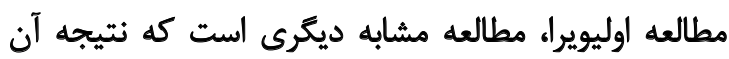

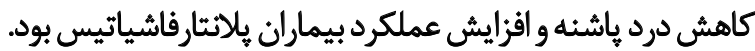

20. Cavus

21. Metatarsal Pad

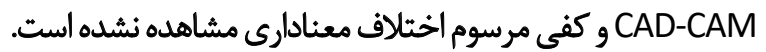

ث

هر دو نوع كفى مطالعه ما موجب بهيبودى درد، فعاليتهاى

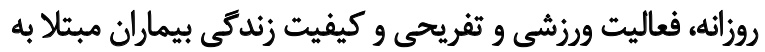

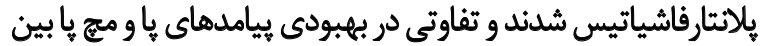

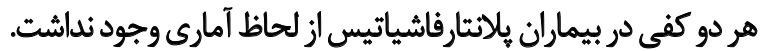

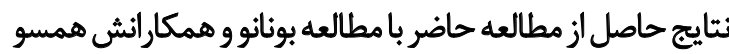

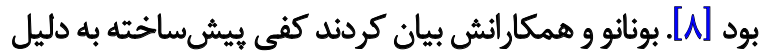

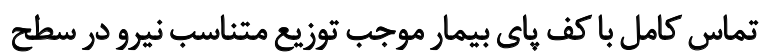

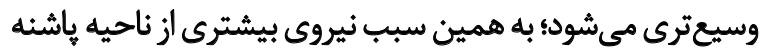

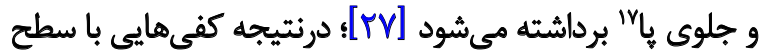

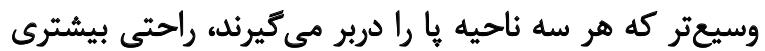

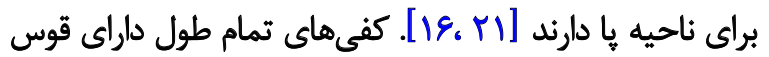

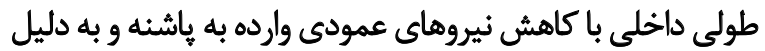

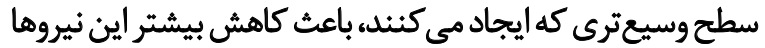

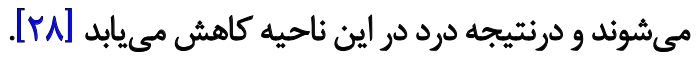

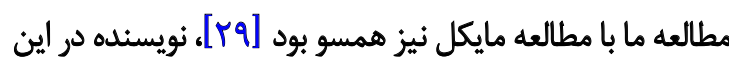

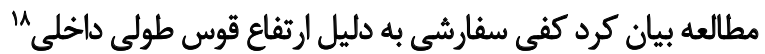

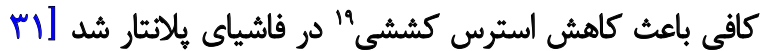

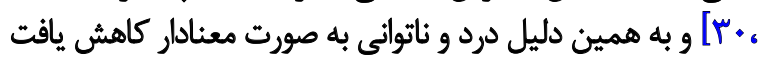

19. Strain Force 
در مطالعه اوليوويراو همكارانش كفى سفارشى ساخت به دليل

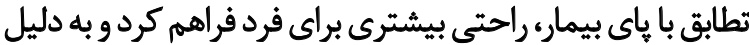

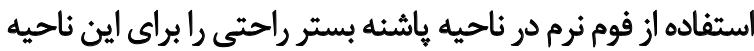

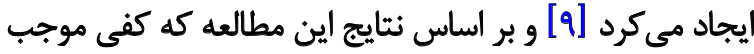

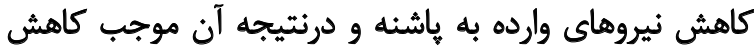

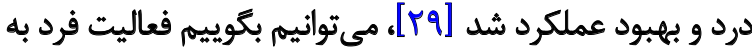

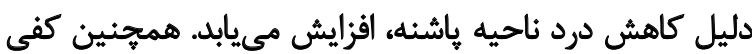

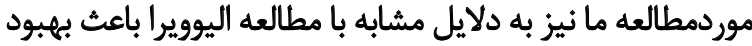
عملكرد و درنتيجه افزايش فعاليت فرد مي شودائ در مطالعه روس و همكارانش، كفى سفارشىساخت انتخاب

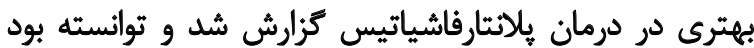

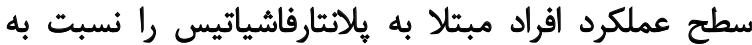
اسيلينت شبانه بالاتر ببرد [هاد].

در مطالعه دريك و همكارانش، استفاده از كفى سفارشى به

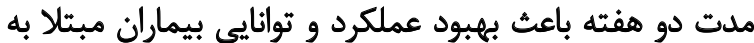

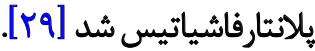

در مطالعه ميشل و همكارانش، كفى سفارشىساخت بعد از

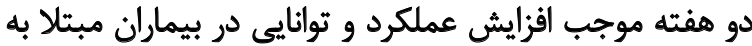

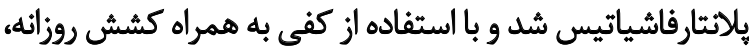

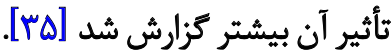

در مطالعه روزنبرى و همكارانش، كفى سفارشىساخت

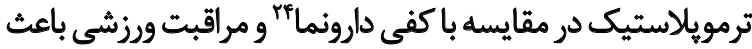

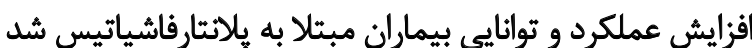

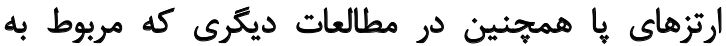

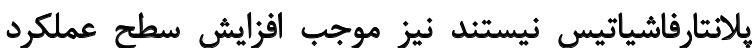

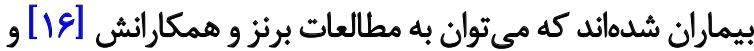

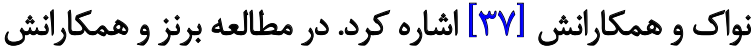

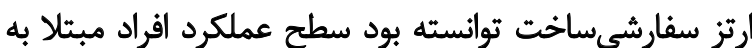

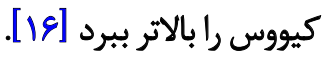

نواك و همكارانش نيز به اين نتيجه رسيدند كه ارتزهاى

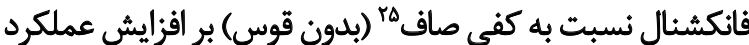

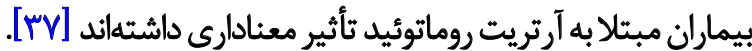

نتايج مطالعه حاضر با مطالعات ذكرشده در بالا مطابقت داشت.

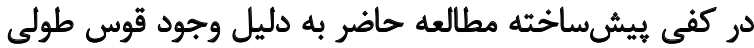

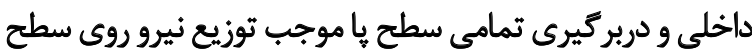

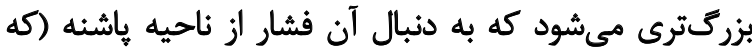

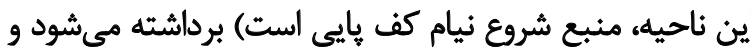

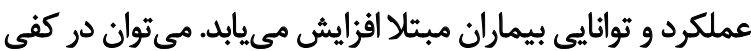

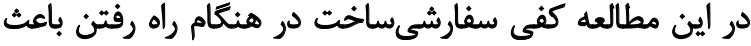

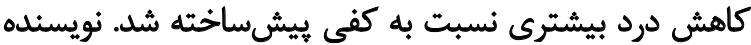

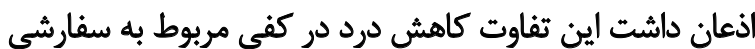

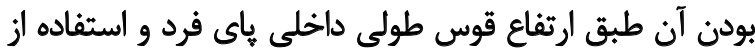

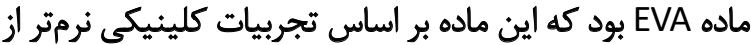

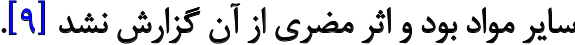
نتايج حاصل از مطالعه اوليويرا همسو با مطالعه حاضر است

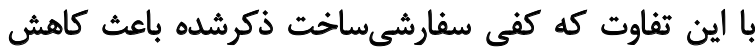

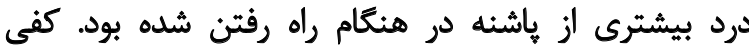

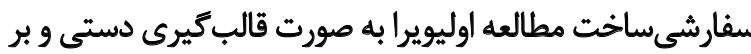

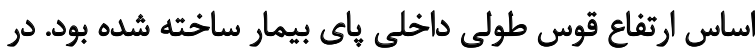

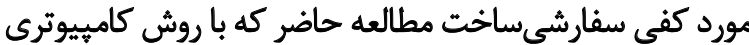

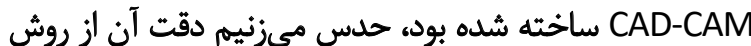
دستى در اين مورد كمتر باشد.

نتيجه مطالعه حاضر با مطالعه بفيفر، ناهمسو بود، در مطالعه كلن

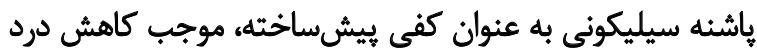

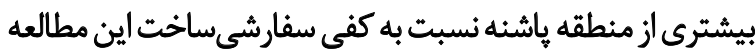

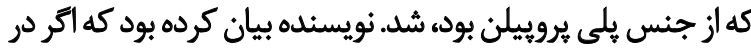

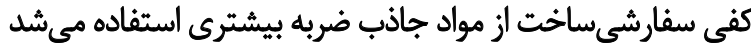

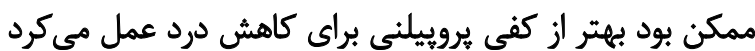

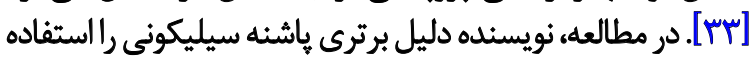

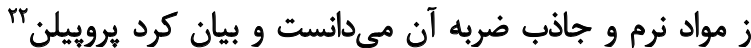

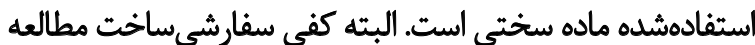

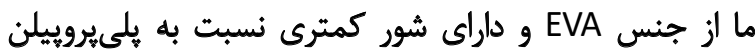

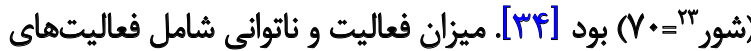
روزانه و فعاليتهاى ورزشى و تفريحى است.

در زمينه فعاليتهاى روزانه و فعاليتهاى ورزشى و تفريحى

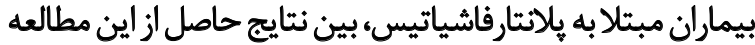

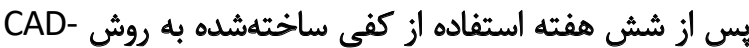

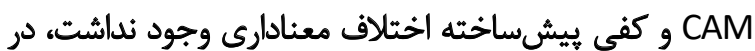

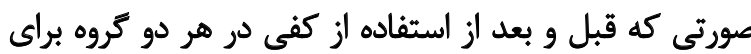

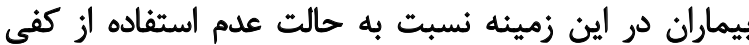

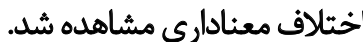

از لحاظ تأثير و مقايسه ارتزها بر روى فعاليتهاى روزانه بان إين

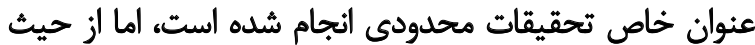

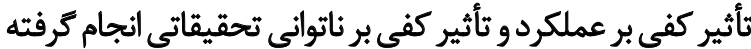

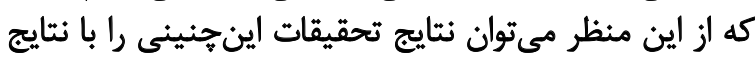

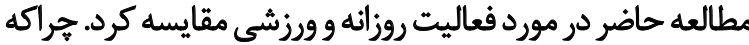

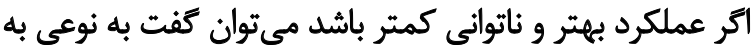

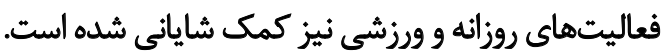




\section{نتيجهيَيرى}

هر دو كفى در بهبود تمام خردهمقياسهاى يرسشنامه

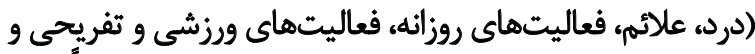

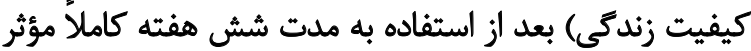

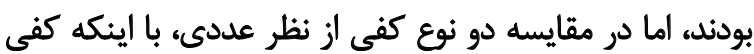

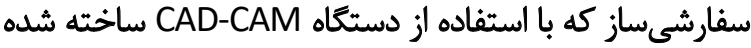

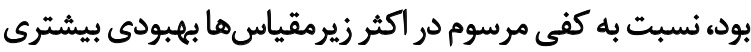

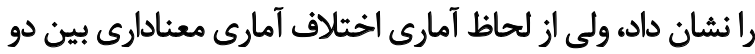

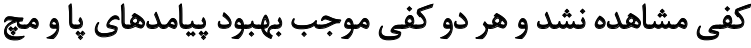

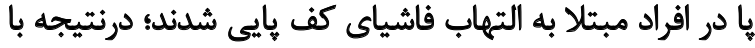

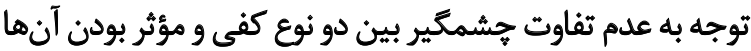

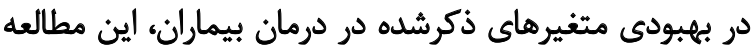

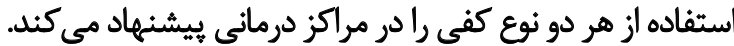

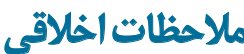 \\ بيروى از اصول اخلاق يؤهش}

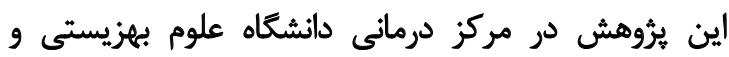

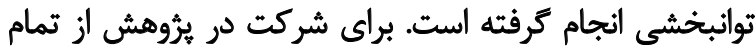

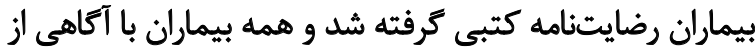

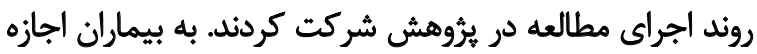

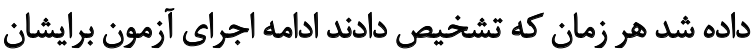

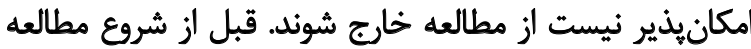

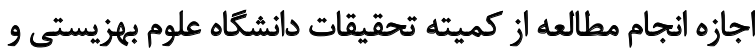
توانبخشى ترفته شد.

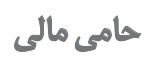

اين مطالعه از بايان نامه كارشناسى ارشد نويسنده اول در كروه

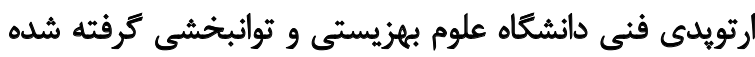

$$
\text { مشاركت ثويسند متان }
$$

تمامى نويسندكًان در نكارش اين مقاله مشاركت داشتهاند.

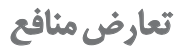

بنابر اظهار نوييسندكان، اين مقاله تعارض منافع ندارد.

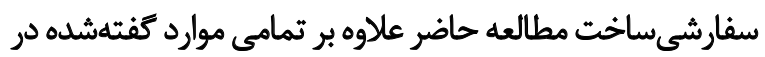

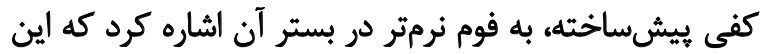
مورد، تطابق بهترى به وجود مى آورد.

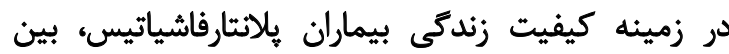

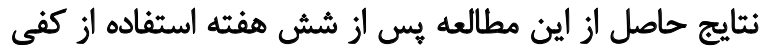

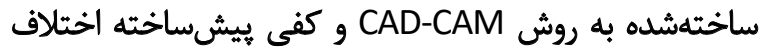

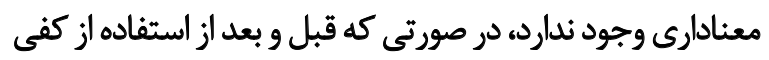

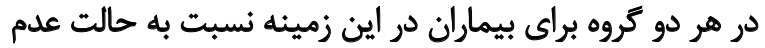

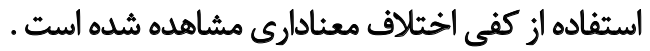

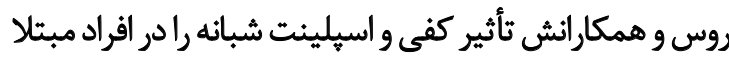

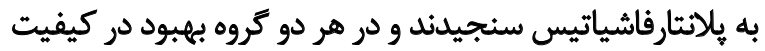

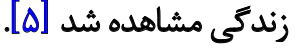
در مطالعه برنز، ارتز موجب بهبودي در كيفيت زندكى افرئ افراد

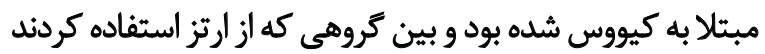

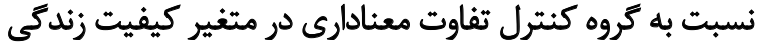

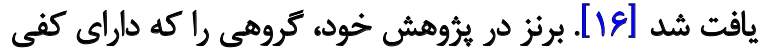

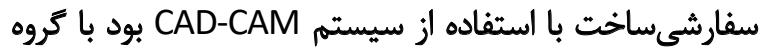

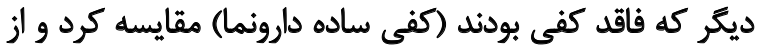

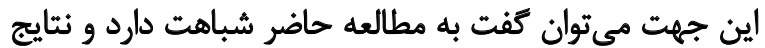

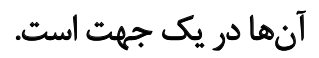

در مورد مطالعات مختلف ذكرشده در اين مبحث، نبايد اين

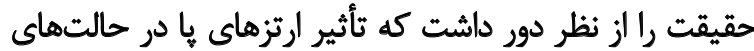

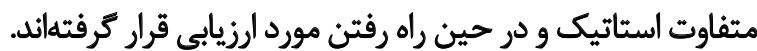

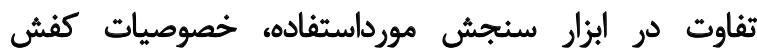

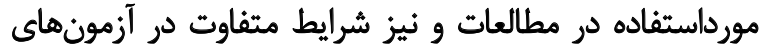

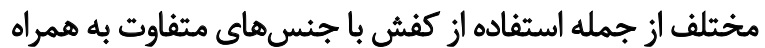

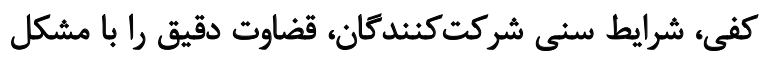

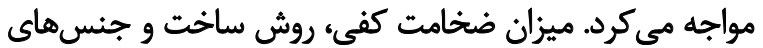

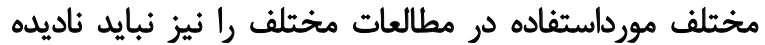

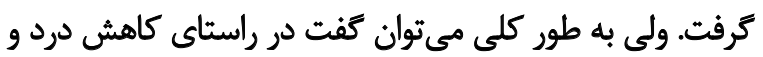

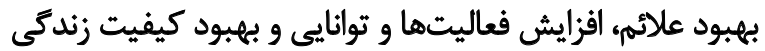
همسو با مطالعات ديكر ارتزهاى اين مطالعه توانستهاند تأثير تواتيرات مطلوبى از خود بروز دهند. به دليل اينكه اين مقاله بر اساس نتايج باياننامه نوشته شده

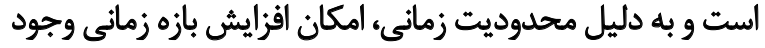

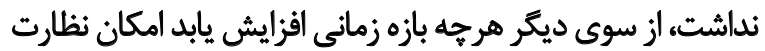

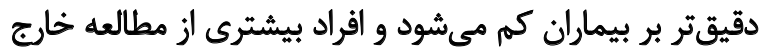

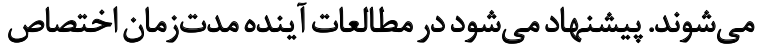

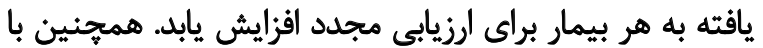

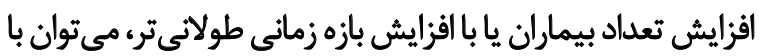
اطمينان بيشترى از نتايج آزمون دفاع كرد. 


\section{References}

[1] Healey K, Chen K. Plantar fasciitis: Current diagnostic modalities and treatments. Clinics in Podiatric Medicine and Surgery. 2010; 27(3):369-80. [DOI:10.1016/j.cpm.2010.03.002] [PMID]

[2] Thomas JL, Christensen JC, Kravitz SR, Mendicino RW, Schuberth JM, Vanore JV, et al. The diagnosis and treatment of heel pain: a clinical practice guideline-revision 2010. The Journal of Foot and Ankle Surgery. 2010; 49(3):S1-S19. [DOI:10.1053/i. jfas.2010.01.001] [PMID]

[3] Nakha'ei Ms, Tahmasbi MN, Karimlou M, Vahab-Kashani R. [Comparison of three orthoses effects on planter fascitis (Persian)]. Archives of Rehabilitation. 2008; 9(3):8-15. https://www. researchgate.net/publication/

[4] Hawke F, Burns J, Radford JA, Du Toit V. Custom-made foot orthoses for the treatment of foot pain. The Cochrane Library; 2008. [DOI:10.1002/14651858.CD006801.pub2] [PMID]

[5] Roos E, Engström M, Söderberg B. Foot orthoses for the treatment of plantar fasciitis. Foot \& Ankle International. 2006; 27(8):606-11. [DOI:10.1177/107110070602700807] [PMID]

[6] Martin JE, Hosch JC, Goforth WP, Murff RT, Lynch DM, Odom $\mathrm{RD}$. Mechanical treatment of plantar fasciitis: A prospective study. Journal of the American Podiatric Medical Association. 2001; 91(2):55-62. [DOI:10.7547/87507315-91-2-55] [PMID]

[7] Chia JK, Suresh S, Kuah A, Ong JL, Phua JM, Seah AL. Comparative trial of the foot pressure patterns between corrective orthotics, formthotics, bone spur pads and flat insoles in patients with chronic plantar fasciitis. Annals Academy of Medicine Singapore. 2009; 38(10):869-75. https://d1wqtxts1xzle7.cloudfront. net/34666914/V38N10p869.pdf?

[8] Bonanno DR, Landorf KB, Menz HB. Pressure-relieving properties of various shoe inserts in older people with plantar heel pain. Gait \& Posture. 2011; 33(3):385-9. [DOI:10.1016/j.gaitpost.2010.12.009] [PMID]

[9] Oliveira HA, Jones A, Moreira E, Jennings F, Natour J. Effectiveness of total contact insoles in patients with plantar fasciitis. The Journal of Rheumatology. 2015; 42(5):870-8. [DOI:10.3899/ jrheum.140429] [PMID]

[10] Telfer S, Woodburn J. The use of 3D surface scanning for the measurement and assessment of the human foot. Journal of Foot and Ankle Research. 2010; 3(1):19. [DOI:10.1186/1757-1146-319] [PMID] [PMCID]

[11] Chen WP, Ju CW, Tang FT. Effects of total contact insoles on the plantar stress redistribution: A finite element analysis. Clinical Biomechanics. 2003; 18(6):S17-S24. [DOI:10.1016/S02680033(03)00080-9]

[12] Bus SA, Ulbrecht JS, Cavanagh PR. Pressure relief and load redistribution by custom-made insoles in diabetic patients with neuropathy and foot deformity. Clinical Biomechanics. 2004; 19(6):629-38. [DOI:10.1016/j.clinbiomech.2004.02.010] [PMID]

[13] Mueller MJ, Lott DJ, Hastings MK, Commean PK, Smith KE, Pilgram TK. Efficacy and mechanism of orthotic devices to unload metatarsal heads in people with diabetes and a history of plantar ulcers. Physical Therapy. 2006; 86(6):833-42. [DOI:10.1093/ptj/86.6.833] [PMID]
[14] Anggoro PW, Tauviqirrahman M, Jamari J, Bayuseno A, Bawono B, Avelina M. Computer-aided reverse engineering system in the design and production of orthotic insole shoes for patients with diabetes. Cogent Engineering. 2018; 5(1):1470916. [DOI:10.1080/ 23311916.2018.1470916

[15] Yurt Y, Şener G, Yakut Y. The effect of different foot orthoses on pain and health related quality of life in painful flexible flat foot: a randomized controlled trial. European Journal of Physical and Rehabilitation Medicine. 2019; 55(1):95-102. [DOI:10.23736/S19739087.18.05108-0] [PMID]

[16] Burns J, Crosbie J, Ouvrier R, Hunt A. Effective orthotic therapy for the painful cavus foot: A randomized controlled trial. Journal of the American Podiatric Medical Association. 2006; 96(3):20511. [DOI:10.7547/0960205]] [PMID]

[17] Ki S, Leung A, Li A. Comparison of plantar pressure distribution patterns between foot orthoses provided by the CAD-CAM and foam impression methods. Prosthetics and Orthotics International. 2008; 32(3):356-62. [DOI:10.1080/03093640802016159] [PMID]

[18] Fong DTP, Pang KY, Chung MML, Hung ASL, Chan KM Evaluation of combined prescription of rocker sole shoes and custom-made foot orthoses for the treatment of plantar fasciitis. Clinical Biomechanics. 2012; 27(10):1072-7. [DOI:10.1016/j.clinbiomech.2012.08.003] [PMID]

[19] Prichasuk S, Subhadrabandhu T. The relationship of pes planus and calcaneal spur to plantar heel pain. Clinical Orthopaedics and Related Research. 1994; (306):192-6. [PMID]

[20] Bijur PE, Silver W, Gallagher EJ. Reliability of the visual analog scale for measurement of acute pain. Academic Emergency medicine. 2001; 8(12):1153-7. [DOI:10.1111/j.1553-2712.2001. tb01132.x] [PMID]

[21] Hong WH, Lee YH, Chen HC, Pei YC, Wu CY. Influence of heel height and shoe insert on comfort perception and biomechanical performance of young female adults during walking. Foot \& Ankle International. 2005; 26(12):1042-8. [DOI:10.1177/1071100705026 01208] [PMID]

[22] Negahban H, Mazaheri M, Salavati M, Sohani SM, Askari M, Fanian $\mathrm{H}$, et al. Reliability and validity of the foot and ankle outcome score: A validation study from Iran. Clinical Rheumatology. 2010; 29(5):479-86. [DOI:10.1007/s10067-009-1344-3] [PMID ]

[23] Huang P, Liang M, Ren F. Assessment of long-term badminton experience on foot posture index and plantar pressure distribution. Applied Bionics and Biomechanics. 2019; 2019:8082967. [DOI:10.1155/2019/8082967] [PMID] [PMCID]

[24] Guldemond N, Leffers P, Schaper N, Sanders A, Nieman F, Willems $\mathrm{P}$, et al. The effects of insole configurations on forefoot plantar pressure and walking convenience in diabetic patients with neuropathic feet. Clinical Biomechanics. 2007; 22(1):81-7. [DOI:10.1016/j.clinbiomech.2006.08.004] [PMID]

[25] Lin TL, Sheen HM, Chung CT, Yang SW, Lin SY, Luo HJ, et al. The effect of removing plugs and adding arch support to foam based insoles on plantar pressures in people with diabetic peripheral neuropathy. Journal of Foot and Ankle Research. 2013; 6(1):29. [DOI:10.1186/1757-1146-6-29] [PMID] [PMCID] 
[26] Hastings MK, Mueller MJ, Pilgram TK, Lott DJ, Commean PK, Johnson JE. Effect of metatarsal pad placement on plantar pressure in people with diabetes mellitus and peripheral neuropathy. Foot \& Ankle International. 2007; 28(1):84-8. [DOI:10.3113/ FAI.2007.0015] [PMID]

[27] Luke BD. Plantar fasciitis: A new experimental approach to treatment. Medical Hypotheses. 2002; 59(1):95-7. [DOI:10.1016/ S0306-9877(02)00120-2]

[28] Menz HB, Zammit GV, Landorf KB, Munteanu SE. Plantar calcaneal spurs in older people: Longitudinal traction or vertical compression? Journal of Foot and Ankle Research. 2008; 1(1):7. [DOI:10.1186/1757-1146-1-7] [PMID] [PMCID]

[29] Gross MT, Byers JM, Krafft JL, Lackey EJ, Melton KM. The impact of custom semirigid foot orthotics on pain and disability for individuals with plantar fasciitis. Journal of Orthopaedic \& Sports Physical Therapy. 2002; 32(4):149-57. [DOI:10.2519/ jospt.2002.32.4.149] [PMID]

[30] Kitaoka HB, Luo ZP, An KN. Analysis of longitudinal arch supports in stabilizing the arch of the foot. Clinical Orthopaedics and Related Research. 1997; (341):250-6. [DOI:10.1097/00003086199708000-00036]

[31] Kogler G, Solomonidis S, Paul J. Biomechanics of longitudinal arch support mechanisms in foot orthoses and their effect on plantar aponeurosis strain. Clinical Biomechanics. 1996; 11(5):243-52. DOI:10.1016/0268-0033(96)00019-8

[32] Burns J, Crosbie J, Hunt A, Ouvrier R. The effect of pes cavus on foot pain and plantar pressure. Clinical Biomechanics. 2005; 20(9):877-82. [DOI:10.1016/j.clinbiomech.2005.03.006] [PMID]

[33] Pfeffer G, Bacchetti P, Deland J, Lewis A, Anderson R, Davis $\mathrm{W}$, et al. Comparison of custom and prefabricated orthoses in the initial treatment of proximal plantar fasciitis. Foot \& Ankle International. 1999; 20(4):214-21. [DOI:10.1177/107110079902 000402] [PMID]

[34] Nitz H, Reichert P, Römling H, Mülhaupt R. Influence of compatibilizers on the surface hardness, water uptake and the mechanical properties of poly (propylene) wood flour composites prepared by reactive extrusion. Macromolecular Materials and Engineering. 2000; 276(1):51-8. DDOI:10.1002/(SICI)14392054(20000301)276:13.0.CO;2-Z]

[35] Drake M, Bittenbender C, Boyles RE. The short-term effects of treating plantar fasciitis with a temporary custom foot orthosis and stretching. Journal of Orthopaedic \& Sports Physical Therapy. 2011; 41(4):221-31. [DOI:10.2519/jospt.2011.3348] [PMID]

[36] Rasenberg N, Fuit L, Poppe E, Kruijsen-Terpstra A, Gorter K, Rathleff M, et al. The STAP-study: The (cost) effectiveness of custom made orthotic insoles in the treatment for plantar fasciopathy in general practice and sports medicine: Design of a randomized controlled trial. BMC Musculoskeletal Disorders. 2016; 17(1):31. [DOI:10.1186/s12891-016-0889-y] [PMID] [PMCID]

[37] Novak P, Burger H, Tomsic M, Marincek C, Vidmar G. Influence of foot orthoses on plantar pressures, foot pain and walking ability of rheumatoid arthritis patients-a randomised controlled study. Disability and Rehabilitation. 2009; 31(8):638-45. [DOI:10.1080/09638280802239441] [PMID] 